\title{
CO $J=3-2$ mapping of the molecular circumnuclear disk in Centaurus A
}

\author{
H. Liszt* \\ National Radio Astronomy Observatory, 520 Edgemont Road, Charlottesville, VA 22903-2475, USA \\ Received 26 January 2001 / Accepted 21 March 2001

\begin{abstract}
We have mapped the molecular circumnuclear disk in Centaurus A in the $J=3-2$ line of ${ }^{12} \mathrm{CO}$ using the James Clerk Maxwell Telescope at $14^{\prime \prime}$ (235 pc) resolution. The disk remains at best only very barely resolved and even the most basic conclusions regarding its structure are highly model-dependent.
\end{abstract}

Key words. elliptical galaxies - molecular gas

\section{Introduction}

Neutral gas in the nearby elliptical NGC 5128/Centaurus A has been studied extensively since the original detection of extragalactic H I absorption by Roberts (1970). On kpc scales in the dust lane, H I (van Gorkom et al. 1990), CO (Phillips et al. 1987; Quillen et al. 1992) and $\mathrm{H} \alpha$ (Bland et al. 1987; Nicholson et al. 1992) all partake of the same behaviour and can be used in concert to construct more robust models. For instance, Eckart et al. (1999) recently showed that the $\mathrm{H} \alpha$ model of Nicholson et al. (1992) can be extended to explain the (mostly red-shifted) atomic and molecular absorption (Israel et al. 1990, 1991; Wiklind \& Combes 1997) as arising in high-altitude gas in the outlying dust lane. Taken together, the observations support the basic picture of a heavily-warped, rotating and precessing disk of gas, presumably captured during the ingestion of a hapless disk galaxy at some time in the past (Baade \& Minkowski 1954; Tubbs 1980; van Gorkom et al. 1990; Quillen et al. 1993).

On the smallest spatial scales accessible in emission in neutral gas - until recently, a few hundred pc $\left(1^{\prime \prime}=\right.$ $16.97 \mathrm{pc}$ at the assumed distance of Cen A, 3.5 Mpc) - the existence of a small molecular circumnuclear disk (cnd) had been inferred from the presence of spatiallyunresolved broad wings on CO profiles (Israel et al. 1990, 1991; Rydbeck et al. 1993). The cnd is entirely absent in $\mathrm{H} \alpha$ but is seen as a weak, extended component of the nuclear sub-mm continuum (Hawarden et al. 1993) at $800 \mu \mathrm{m}$ and $450 \mu \mathrm{m}$.

The diameter of the molecular cnd has variously been inferred by deconvolution (Rydbeck et al. 1993) and excitation analysis (the work of Israel and collaborators) to range from 200 to 500 pc. Rydbeck et al. (1993), who

\footnotetext{
* e-mail: hliszt@nrao.edu
}

used the highest spatial resolution (375 pc) to date to map $\mathrm{CO}$ in the cnd, found the smaller values for the radius of the disk and showed that the position angle of the cnd is substantially higher than that of the dust lane, which is $\approx 120^{\circ}$ : the cnd is aligned perpendicular to the base of the radio jet emanating from the core in this famous double radio source. The sub-mm continuum is also extended along similar position angles $140^{\circ}-145^{\circ}$. Recently, $\mathrm{H}_{2}$ emission has been observed along several position angles by Marconi et al. (2001) at $\lambda 2.12 \mu$ using the VLT; recombination lines from ionized gas in the same waveband show only the contributions of gas in the dust lane.

Here we present the results of mapping CO $J=3-2$ emission toward the inner regions of the Cen A dust lane using the $14^{\prime \prime}$ beam (235 pc) of the JCMT. Our basic observational and modelling techniques are discussed in Sect. 2. The results are presented and modelled in Sect. 3, where we also discuss some implications of our work for somewhat larger-scale models of the dust lane. These have probably been compromised slightly by the relatively low velocity resolution of the $\mathrm{H} \alpha$ data from which the most detailed picture of the dust-lane has been constructed. Section 4 is a brief summary of our conclusions.

\section{Observing and modelling}

\subsection{At last, data}

The observations were done in the $J=3-2$ line of ${ }^{12} \mathrm{CO}$ (rest frequency $345.80 \mathrm{GHz}$ ) by Goeran Sandell during Dutch service-observing time in 1997 February; this project was originally begun in 1988 May, but five trips to the telescope were totally ruined by weather and, earlyon, equipment and teething problems. The weather during this observing was truly excellent, with system temperatures in the range 400-450 K; unfortunately only one 


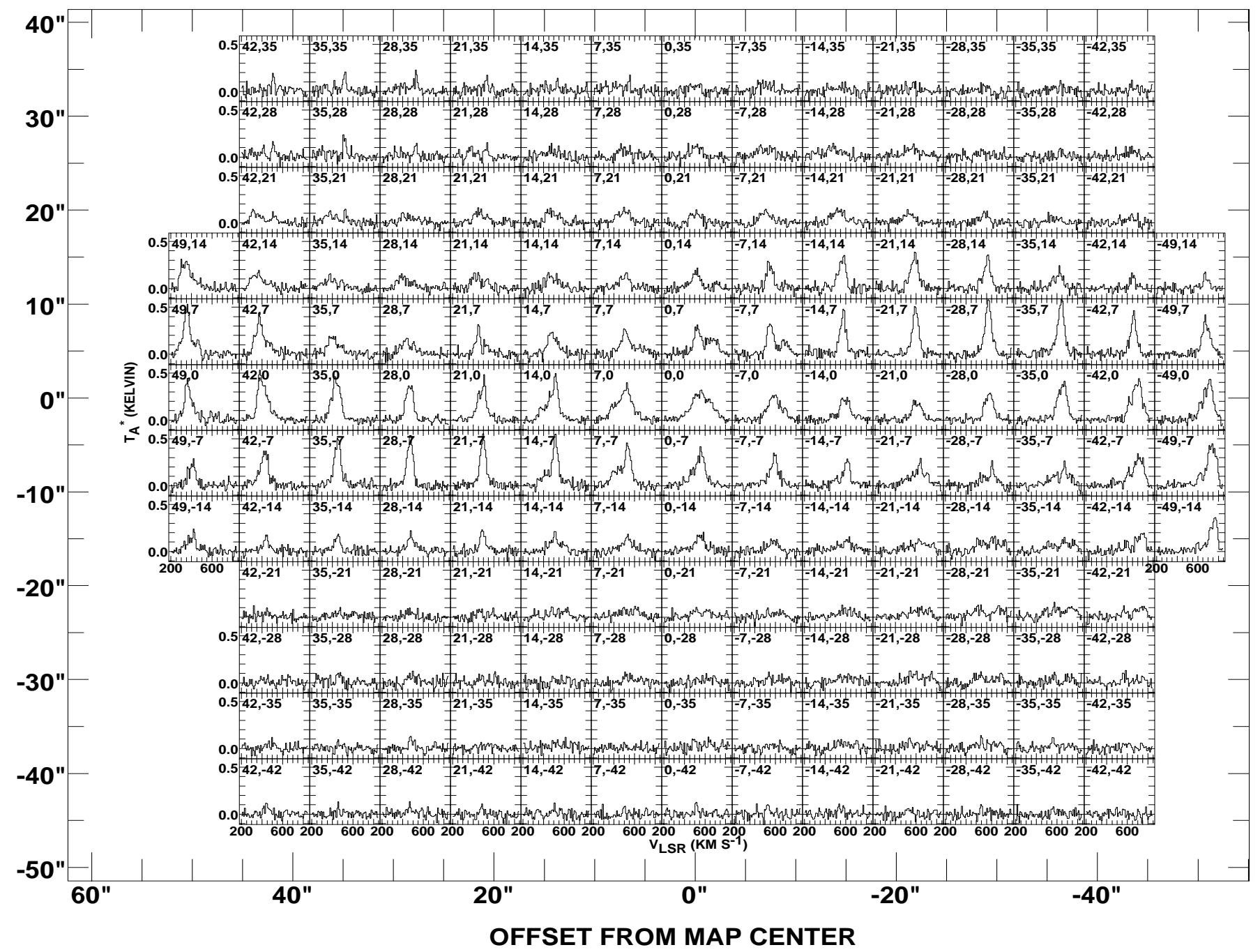

Fig. 1. Observed ${ }^{12} \mathrm{CO}$ CO $J=3-2$ spectra in Centaurus A. The horizontal axis of this $7^{\prime \prime}$ grid of spectra is in position angle $\Omega=116.5^{\circ}$ on the sky

channel of the receiver was working. We smoothed the original data down to a resolution and channel separation of $3.26 \mathrm{~km} \mathrm{~s}^{-1}$ to achieve a typical channel-to-channel rms of $0.055 \mathrm{~K}$. The spectra cover the range $V_{\mathrm{LSR}}=133 \mathrm{~km} \mathrm{~s}^{-1}$ to $947 \mathrm{~km} \mathrm{~s}^{-1}$ centered at $V_{\mathrm{LSR}}=540 \mathrm{~km} \mathrm{~s}^{-1}\left(V_{\mathrm{HEL}}=\right.$ $V_{\mathrm{LSR}}+2.5 \mathrm{~km} \mathrm{~s}^{-1}$ and $\left.V_{\mathrm{OPTICAL}}=V_{\mathrm{RADIO}}+1.0 \mathrm{~km} \mathrm{~s}^{-1}\right)$. The intensity scale of the results is $T_{\mathrm{A}}^{*}=\eta * T_{\mathrm{MB}}$ where $\eta \approx 0.5$. The $H P B W$ of the JCMT is $14.0^{\prime \prime}$ at the frequency of observation, $345.2 \mathrm{GHz}$.

The data were taken by integrating for $1 \mathrm{~min}$ at each point on a grid of sky positions spaced by $7^{\prime \prime}$ and centered at the 1950 coordinates $13^{\mathrm{h}} 22^{\mathrm{m}} 31^{\mathrm{s}} .8,-42^{\circ} 45^{\prime} 30^{\prime \prime}$, oriented along the position angle $\Omega=116.5^{\circ}$ measured East from North. We beam-switched in position angles $\Omega=116.5^{\circ} \pm 90^{\circ}$. In all, 166 spectra were taken as shown in Fig. 1. Figure 2 shows the data in another format where we have generated position-velocity diagrams along strips spaced by $10^{\circ}$ in position angle, using simple bilinear interpolation. In that figure, the position angle on the sky of any strip is $\alpha+116.5^{\circ}$. Figures 4 or 5 show the innermost spectra, containing emission from the cnd, in more detail.
The spectra near the center (Figs. 4 and 5) readily betray an overall 0.5-pixel pointing error to the West when compared with models which are centered on the nucleus; that is to say, the nucleus of Cen A is apparently to be found 0.5 pixels to the East (to the left) of our nominal center along the horizontal axis of the map grid, if the cnd is assumed to be centered on the nucleus. Other pointing errors certainly affect the data but apparently to a lesser extent. Modelling of the data was not greatly improved by allowing for pointing errors on a pixel-by-pixel basis.

\subsection{Models}

To model the kinematics of the data we numerically integrated along lines of sight through fully three-dimensional, plane-parallel stratified disks of optically-thin gas, as described by Liszt (1992). In such models, density and brightness are interchangeable; the physical density is equivalent to a luminosity density. The use of an optically thin approximation to the CO emission is justified on several grounds, even with the nearly edge-on 


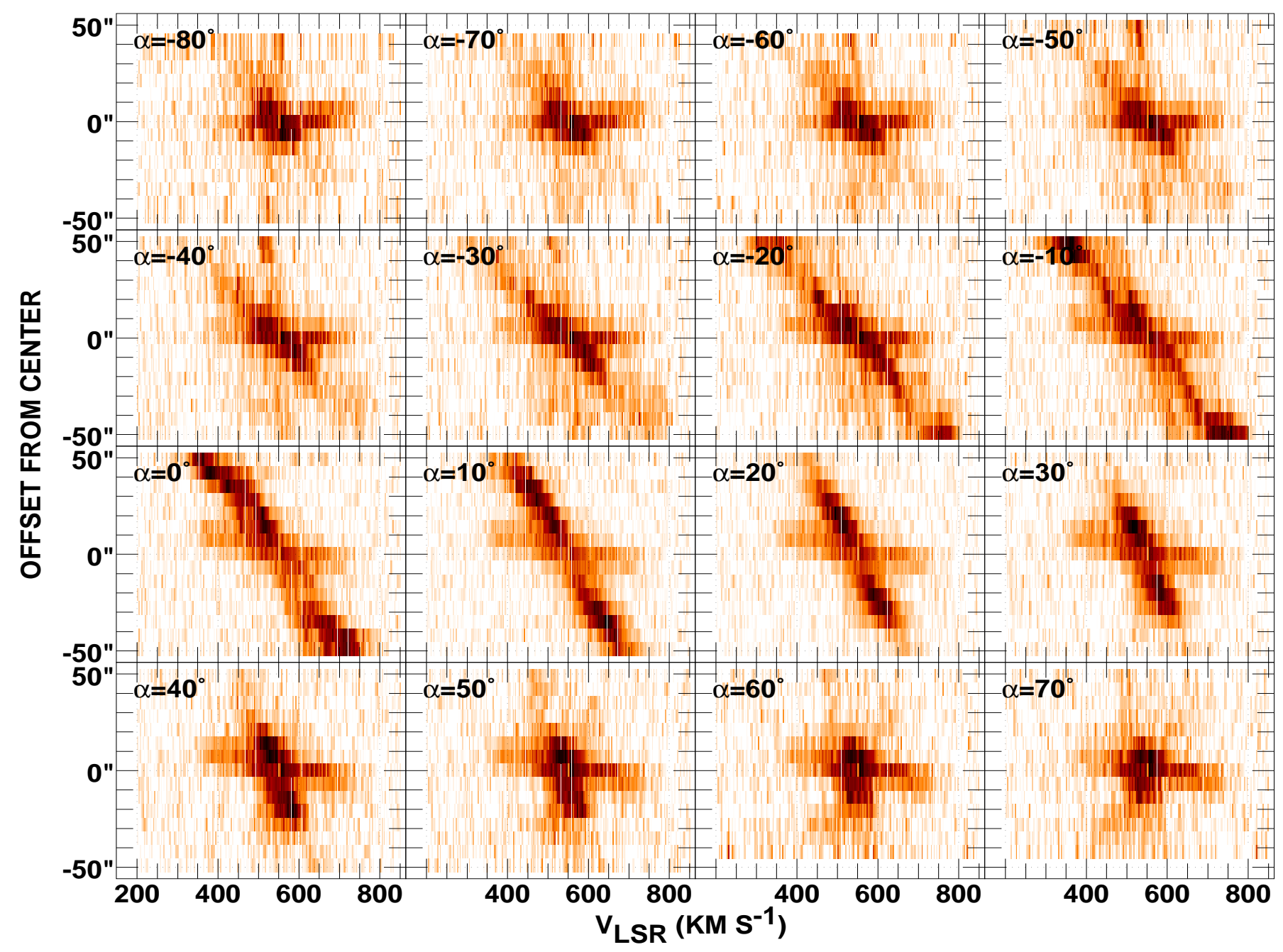

Fig. 2. Position-velocity diagrams formed from the profiles in Fig. 1 using bilinear interpolation. The position angle on the sky is $\alpha+116.5^{\circ}$ and positive displacements are to the left of the grid's midpoint as shown in Fig. 1

geometry found for the cnd. Prior analyses of CO excitation in the cnd by Israel et al. $(1990,1991)$ and Israel (1992) found mean excitation temperatures of some $25 \mathrm{~K}$ up to the $J=3-2$ transition, compared to the typical observed antenna temperatures of $\lesssim 0.3 \mathrm{~K}$ seen here. Because our models fill an appreciable fraction of the $14^{\prime \prime}$ main beam (about 2/3 for the $v \propto r$ model described in Sect. 3.2), it follows that the low observed intensities are not the result of beam dilution. Instead there are two explanations; the gas can be spread uniformly over the cnd and be optically thin in the usual sense, or it can be clumped with a low surface-covering factor (in which case the $\mathrm{CO}$ can be either thick or thin in the individual clumps). Either way, little emission is trapped within the cnd and radiative transfer effects are unimportant. Analyses of other molecules (ibid) imply optically thin conditions in those cases as well.

For the cnd, we formed profiles every $0.3^{\prime \prime}$ and convolved these with a $14^{\prime \prime}(H P B W)$ Gaussian sampled over $2 H P B W$ in all directions. For the larger gas disk in the dust lane, profiles were formed every $1.3^{\prime \prime}$ followed by the same convolution. Models ran in a few tens of seconds on a $550 \mathrm{MHz}$ Pentium computer, with the innermost loops hand-coded in assembly language for the coprocessor.

To fit the models of the cnd, we used the wings of the profiles and normalized the aggregate integrated intensity in the models to the same sum in the data, then did a least-squares minimization on the channel-by-channel differences between data and model. In fact the basics of the model were established almost immediately by inspection but the least-squares technique was a simple way of refining the models and discovering those parameters to which the observations were most sensitive.

Input to the models requires specifying the radial variation of position angle, inclination, gas surface density, Gaussian vertical dispersion, velocity dispersion, and rotation velocity. Except for the gas surface density and rotation velocity, all of these were kept constant with radius; all models we made of the cnd were for a flat (unwarped) disk. Because the red-shifted absorption seen toward the nucleus can be explained by gas at larger radii in the dust lane (see below) all of the modelling was done for pure rotational motion. The center velocity is an adjustable parameter, as is the pointing error but the latter was obvious 
from inspection of the profiles and was fixed at 0.5 pixel along the position angle of the horizontal axis of the map grid.

There is some ambiguity in the models in that emission near the systemic velocity may arise either from the cnd or from more distant portions of the dust lane seen in projection; this is discussed further at the end of Sect. 3.2. For this reason, we found it of interest to model the warp in the dust lane as well. No least squares minimization was attempted and no definitive global modelling is possible given the limited extent of our data. But some suggested alterations in extant models of the dust lane are easily inferred from comparisons with our profiles.

\subsection{Cores and kinematics in elliptical galaxies}

It has become apparent in the last few years that all sufficiently bright ellipticals $\left(M_{V}<-20.5\right)$ have stellar cores in which the slope (with radius) of the luminosity density is substantially shallower than that outside (Ferrarese et al. 1994; Lauer et al. 1995; Gebhardt et al. 1996; Lauer et al. 1997; Faber et al. 1997). In many systems, the core size, parametrized in terms of a "break radius" where the transition between inner and outer power laws occurs, is comparable to the size of the Cen A cnd inferred from earlier data. Thus it seemed reasonable, at the time of the observations, that the kinematics of the cnd would probe the core of Cen A, whose properties had always been obscured by dust at optical wavelengths. Events have made this view rather antique.

Cen $\mathrm{A}$ is bright enough to have a core $\left(M_{V}=-21\right.$ from the RC3 or -22 from Marconi et al. 2000) but not so bright that it is in the regime where the core radius is necessarily large or fixed by $M_{V}$. As it turns out, the break radius for Cen $\mathrm{A}$ is only $4^{\prime \prime}-5^{\prime \prime}$ or $70-85 \mathrm{pc}$ (ibid), which is small compared to the previously-determined radii of the cnd, so any identification of the cnd and core is harder to substantiate. Or perhaps more interestingly, the cnd apparently will, with proper resolution, display the kinematics in this interesting transition region.

In the most common current parametrization (Lauer et al. 1995) the slope of the luminosity with projected angular displacement from the core $\theta$ is expressed as a power law $I(\theta) \propto \theta^{-\gamma}(\gamma>0)$ leading to a luminosity density variation with radius proportional to $r^{(-1-\gamma)}$ and a circular velocity $v \propto r^{(1-\gamma) / 2}$ assuming a constant massto-light ratio. For Cen A, $0.0 \leq \gamma \leq 0.3$ (Marconi et al. 2000) leading to $0.5 \geq(1-\gamma) / 2 \geq 0.35$.

Figure 3 shows several rotation laws of relevance to our modelling of Cen A. A representation of the speed variation seen in $\mathrm{H}_{2}$ by Marconi et al. (2001) is given as a piece-wise continuous function (it is this choppy). Also shown is a solid-body rotation curve which is by far the best fit to our data, and a $v \propto r^{0.4}$ law which reaches about the same maximum velocity. The $\mathrm{H}_{2}$ velocity field is not badly fit by the 0.4 power law over much of its extent.

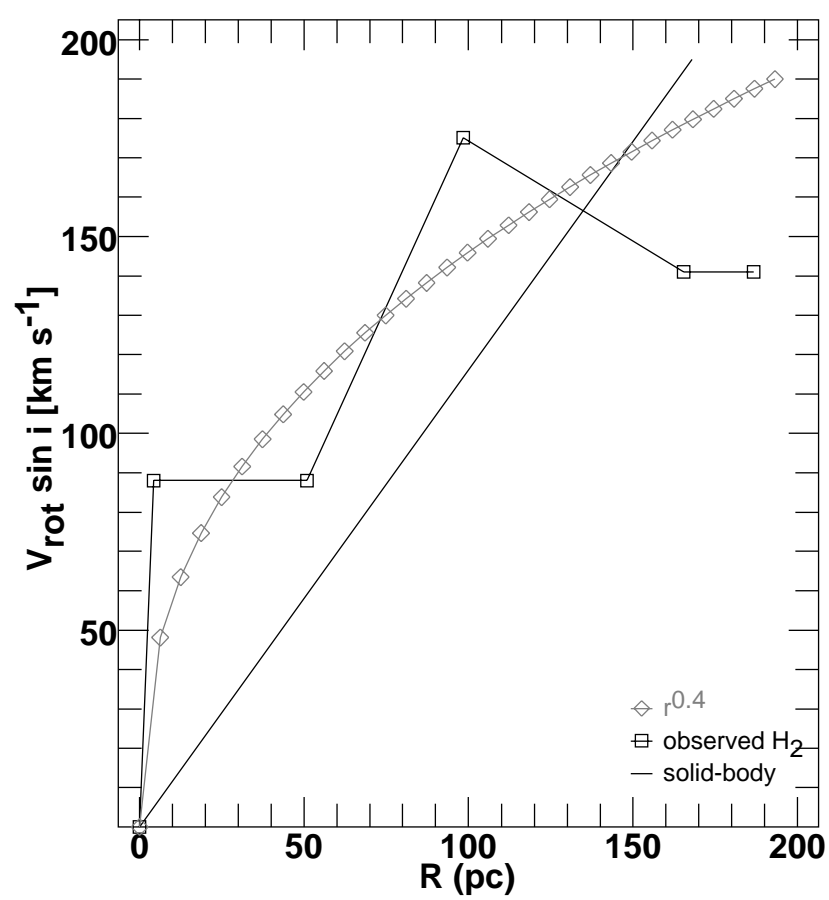

Fig. 3. Rotation curves for cnd models. Shown are two powerlaws and our representation of the velocity variation actually observed in $\mathrm{H}_{2}$ by Marconi et al. (2001)

\section{Basic results and models}

\subsection{Observational results}

The spectra in Figs. 1 and 2 cover about $40 \%$ of the linear extent of $\mathrm{CO}$ emission in the inner, least-warped regions of the dust lane, which reaches outward some $2^{\prime}$ from the nucleus in the maps of Quillen et al. (1992) in position angles near $120^{\circ}$. Our data confirm that the basic kinematics of $\mathrm{CO}$ and $\mathrm{H} \alpha$ are largely identical (with the obvious and very important exception of the molecular cnd). In particular, the strongest velocity gradient over the central $100^{\prime \prime}$ is found along strips with $\alpha \approx-30^{\circ}, \Omega \approx 85^{\circ}$, while the position angle of the central portions of the dust lane is closer to $\Omega=115^{\circ}$, increasing at larger radii. Large-scale mapping of the $\mathrm{CO}$ emission cannot be narrowly confined across the major axis because of this profound warp.

The full extent of the CO emission is not directly known from prior mapping, although it probably follows the $\mathrm{H} \alpha$ quite closely. The weak emission seen in the upper left hand corner of Fig. 1 is quite real and is naturally explained as part of the high- $z$ extension of more distant portions of the dust lane seen in projection. The dustlane models of Nicholson et al. (1992) reproduce it quite directly. In a map of emission near the systemic velocity, this emission contributes to what appears to be a separate component of the gas lying across the dust lane, parallel to the major axis of the optical image of the host galaxy.

The position angle of the major axis of the cnd is noticeably greater than $116.5^{\circ}$ as was earlier inferred by Rydbeck et al. (1993). An interesting distinction between our data and the work of Nicholson et al. (1992) is the 
line splitting which occurs across the entire inner region sampled here along the strips at $\alpha=-10^{\circ}$ and $-20^{\circ}$. In the $\mathrm{H} \alpha$ data, this splitting was remarked only near the endpoints of our strips (Nicholson et al. 1992) most likely because it was not resolved spectroscopically at smaller radii where the velocity separation is smaller. Previous CO observations by Phillips et al. (1987) also showed this splitting only at the extremities of these strips, at least partly because of low spatial resolution. The existence of this splitting over the innermost regions has interesting ramifications for the inferred kpc-scale behaviour of the dust lane.

\subsection{Basic models of the cnd emission}

The best-fitting and simplest model we were able to construct of the cnd is shown superposed on the innermost profiles in Fig. 4. This model is quite simple and perhaps equally implausible; a thin, radially-uniform, edgeon $\left(i=85^{\circ}\right), 168$-pc (radius) disk in solid-body rotation at $195 / \sin i \mathrm{~km} \mathrm{~s}^{-1}$ at its tip. The vertical dispersion is $47 \mathrm{pc}$ and the velocity dispersion is $8 \mathrm{~km} \mathrm{~s}^{-1}$. It fits the data quite well, and perhaps even too well, since we expect a contribution to emission at the systemic velocity from gas at larger radii seen in projection. At least toward the $(0,0)$ position this contribution could in principle be almost nil since the cnd model alone accounts for so much of the observed emission. Emission within $75 \mathrm{~km} \mathrm{~s}^{-1}$ of the systemic velocity was not used in the least-squares minimization by which the model parameters were refined so it is something of a bonus that some models account for so much of it, and perhaps something of a puzzle as well.

There are a variety of strong statements which could be made about the $\mathrm{CO}$ distribution on the basis of this model's ability to account for so much of the emission observed at the central (however slightly eccentric) position; the cnd cannot be too annular nor too strongly dominated by a central massive point-source $\left(M \lesssim 210^{8} M_{\text {sun }}\right)$ or the fit is seriously disturbed. Also, in this parametrization, the disk is resolved (the $20^{\prime \prime}$ diameter is substantially larger than the $H P B W$ of $14^{\prime \prime}$ ), and modelling is more sensitive to many of the input parameters than is the case for the other models discussed here. But faced with the implausibility of a constant-density core, which would seemingly be needed to support solid-body rotation (in equilibrium, at least) and which has never been observed in any of the ellipticals studied to date - we will explore better-founded alternatives.

The cnd was observed in the $\lambda 2.12 \mu$ lines of $\mathrm{H}_{2}$ by Marconi et al. (2001) and the rotation curve from that work is shown here in Fig. 3. Our data can be fit with either the $r^{0.4}$ law shown there or (slightly more poorly) the piecewise continuous velocity field of the $\mathrm{H}_{2}$ data. Shown in Fig. 5 is a fit using the $r^{0.4}$ law with the following other differences from the solid-body, radially-constant cnd model: the nominal radius is increased to $190 \mathrm{pc}$, the gas velocity dispersion is increased to $20 \mathrm{~km} \mathrm{~s}^{-1}$ and the mid-plane luminosity density varies as $\exp \left(-2(r / 190 \mathrm{pc})^{2}\right)$. A strong radial taper is absolutely required by the observing geometry; when the circular velocity rises rapidly, very little of the disk appears near the systemic velocity and the profiles tend to be sharply cusped at their extremities (in velocity) all over the map. The increased velocity dispersion helps to smooth over some of the hornedness of the profiles as well.

In this case, the cnd is not really resolved (the "halfpower" width of the Gaussian luminosity density taper, $220 \mathrm{pc}$, is slightly smaller than the $H P B W$ of the JCMT) and the modelling is considerably less sensitive to many of the input parameters. One comment can however, be made. Marconi et al. (2001) identified the cnd with only that portion of the rotation curve at $r \gtrsim 6^{\prime \prime}$, where the observed rotation speed exhibited a pseudo-Keplerian decline. The CO models really cannot tolerate such a large inner gap, for easily understandable reasons. Given that we had to impose a strong radial taper on the surface density, it follows that we cannot also remove the innermost regions of the disk and maintain the fit. The fit may be preserved in the presence of a slight gap by tapering the luminosity density more strongly, but only for relatively small inner radii, perhaps $30 \mathrm{pc}$ or less.

This points up an interesting difference between our work and the modelling of Rydbeck et al. (1993). By weighting the fit more to the extremities of the line profiles (observed at $22^{\prime \prime}$ resolution in the $J=2-1$ line), they derived a rapidly-rising rotation curve and a smaller, annular gas distribution. The same effect is seen here; the more rapidly rising rotation law requires a smaller structure. The point is that, lacking good spatial resolution, the model that is derived depends strongly on how much of the data is fit. The nature of the fit changes depending on how much of the emission seen close to the systemic velocity is assumed to arise in the cnd. So our picture of the cnd is rather dependent on knowledge of the contributions (emission and absorption) from the dust lane.

\subsection{Where do the inner and outer regions join kinematically?}

Of course, even for solid-body rotation the inherent circular velocity rises much more rapidly with radius in the cnd than is seen over the inner portions of the large-scale dust lane, for instance along the line $\alpha=-30^{\circ}$ in Fig. 2 . The small slope of the main body of gas in that figure was noted previously by Nicholson et al. (1992), since the $\mathrm{CO}$ and $\mathrm{H} \alpha$ show identical behaviour outside the cnd. To explain this behaviour in the $\mathrm{H} \alpha$ light, either (i) the $\mathrm{H} \alpha$ gas must be disposed in an annulus with a large central hole or (ii) the $\mathrm{H} \alpha$ light from the center must be subject to very heavy extinction. The alternative (iii) that the $\mathrm{H} \alpha$ terminal velocity coincides with the circular velocity, and gas occurs nearer the center, requires that the equilibrium circular velocity rise so slowly with radius that it would 


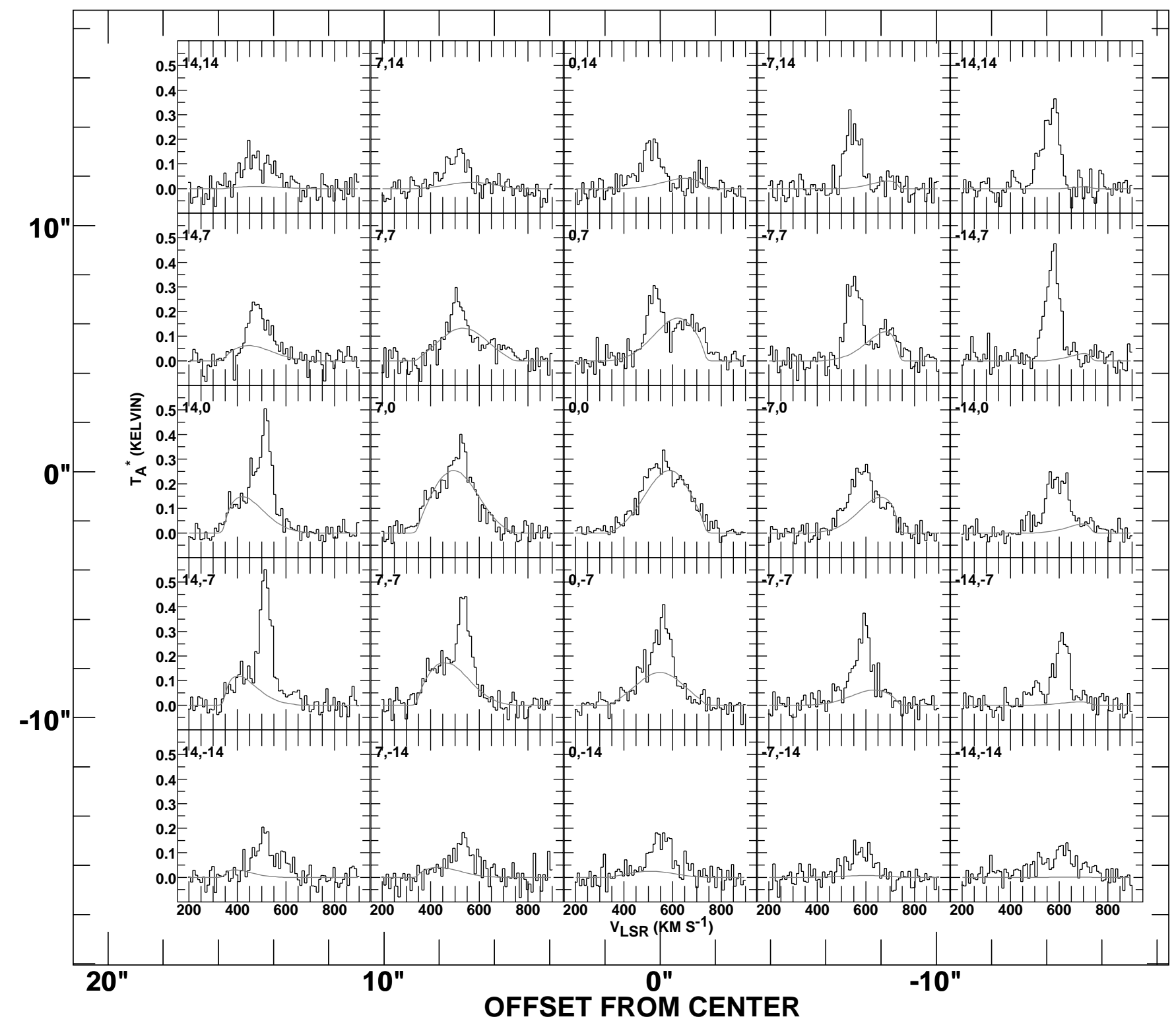

Fig. 4. The innermost spectra from Fig. 1, showing ${ }^{12} \mathrm{CO} \mathrm{CO} J=3-2$ spectra from the circumnuclear disk in Cen A. The horizontal axis of this $7^{\prime \prime}$ grid of spectra is in position angle $\Omega=116.5^{\circ}$ on the sky. Shown superposed on the data are results from our model of a simple, thin, plane-parallel stratified, uniformly emitting (with radius), nearly edge-on circumnuclear disk (cnd) of radius $168 \mathrm{pc}\left(10^{\prime \prime}\right)$ in solid-body rotation. Emission not explained by the cnd originates in more distant portions of the dust lane seen in projection

be very hard to understand with reasonable mass-to-light ratios.

Quillen et al. (1992) made models of the CO emission from the dust lane based on (iii) but our data or the $\mathrm{H}_{2}$ observations show that this is very unlikely. Following Nicholson et al. (1992), and because the CO emission in the dust lane has a large central gap and cannot be similarly extinguished, only (i) seems to be an acceptable alternative.

Marconi et al. (2001) noted that the circular velocity variation seen in the cnd in $\mathrm{H}_{2}$ is inconsistent with the derived luminosity density distribution and they appealed to models of gas bars which have strong non-circular mo- tion; Mirabel et al. (1999) have of course observed a much larger gaseous bar structure in mid-IR light. The redshifted atomic and molecular absorption was at one time viewed as evidence for the cnd (Israel et al. 1990, 1991) and perforce for the existence of strong non-circular motions within it. But, interestingly, it has recently been shown by Eckart et al. (1999) that all of the red-shifted absorption can be explained in the Nicholson et al. (1992) kinematical framework by high-latitude gas further out in the dust lane, at radii of 400-600 pc or more; absorption at the systemic velocity arises even further out in this model. Thus there is no need to incorporate non-circular motions into the cnd for such other purposes. 


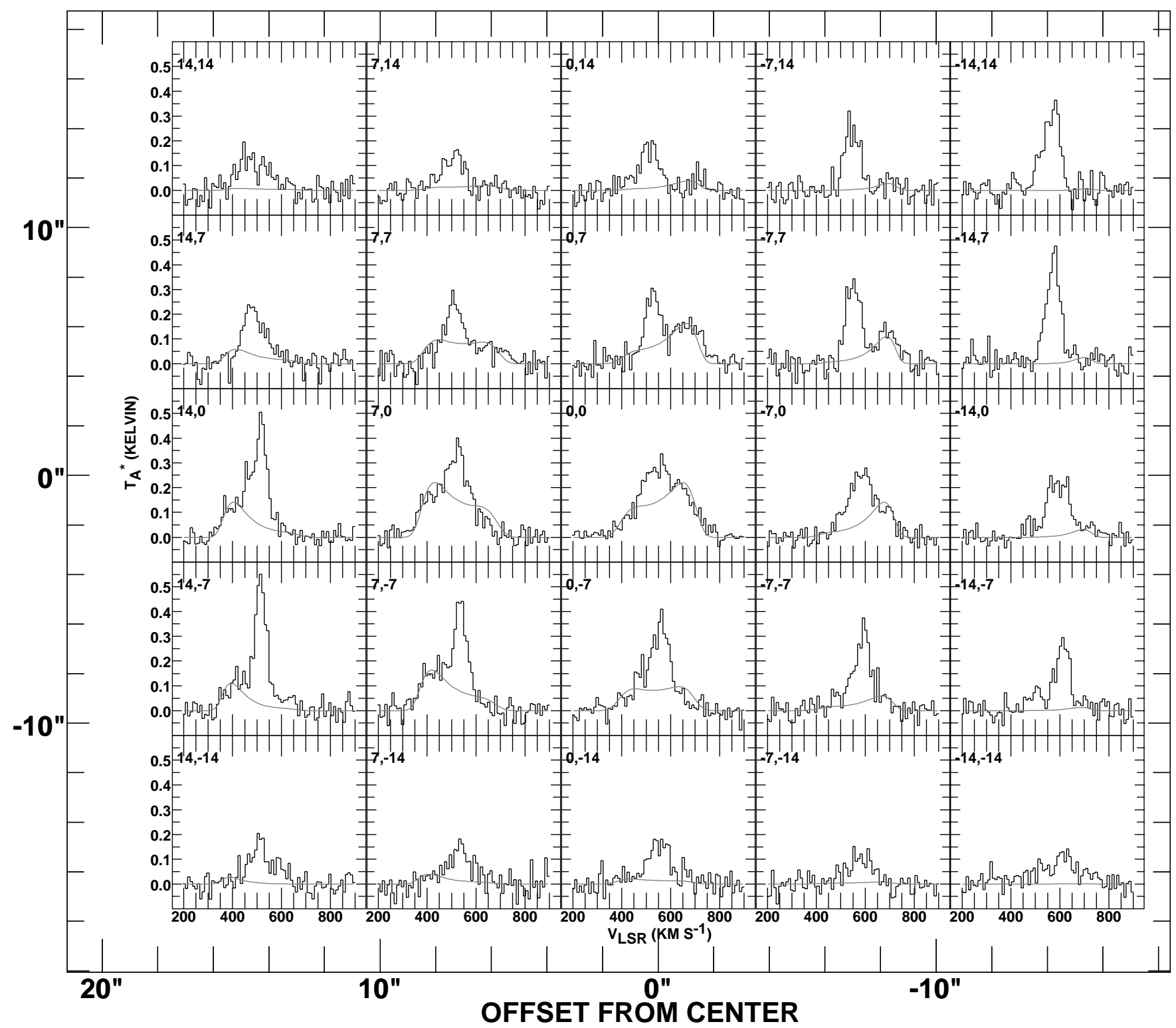

Fig. 5. As in Fig. 4, but for a disk of radius $190 \mathrm{pc}$ rotating as $v \propto r^{0.4}$ and with the surface density varying radially as $\exp \left(-2(r / 190 \mathrm{pc})^{2}\right)$

Somewhere between 200 and $650 \mathrm{pc}$ radius, the circular velocity must increase at least to the observed value of $250 \mathrm{~km} \mathrm{~s}^{-1}$. For the $r^{0.4}$ power-law shown in Fig. 3, the required speed is attained at $r=350 \mathrm{pc}$. The model of Eckart et al. (1999) also requires the attainment of such speeds inside $400-600 \mathrm{pc}$ radius.

\subsection{The influence of absorption on the central profile}

We remarked in Sect. 3.2 that the model which is derived for the cnd depends quite directly on how much of the emission observed nearer the systemic velocity is incorporated. In principle, everything which is not explained by gas in the dust lane should be subsumed by the cnd.

The CO profiles observed near the center are the sum of; emission from the cnd; emission from the projected portions of the outlying dust lane; absorption against the central continuum source by gas in the cnd and dust lane; and absorption of cnd emission by gas in the dust lane. Absorption at $550 \mathrm{~km} \mathrm{~s}^{-1}$ and $605 \mathrm{~km} \mathrm{~s}^{-1}$ is apparent in the centrally-located spectra in Fig. 2 and narrow $\mathrm{CO} J=$ 3-2 absorption components also occur at about $540 \mathrm{~km} \mathrm{~s}^{-1}$ as well (Israel et al. 1991). Gas excited into the $J=2$ level presumably arises in the denser and/or warmer regions along the line of sight.

Figure 6 shows our centermost emission profile along with $v \propto r$ and $v \propto r^{0.4}$ emission models including contributions from the dust lane (see the following section). Also shown are $\mathrm{HCO}^{+}$absorption from Wiklind \& Combes (1997) and $\mathrm{OH}(1667 \mathrm{MHz})$ absorption from van Langevelde et al. (1995). The dust lane kinematics may be extended in the manner of Eckart et al. (1999) to produce the observed red-shifted absorption, but the 


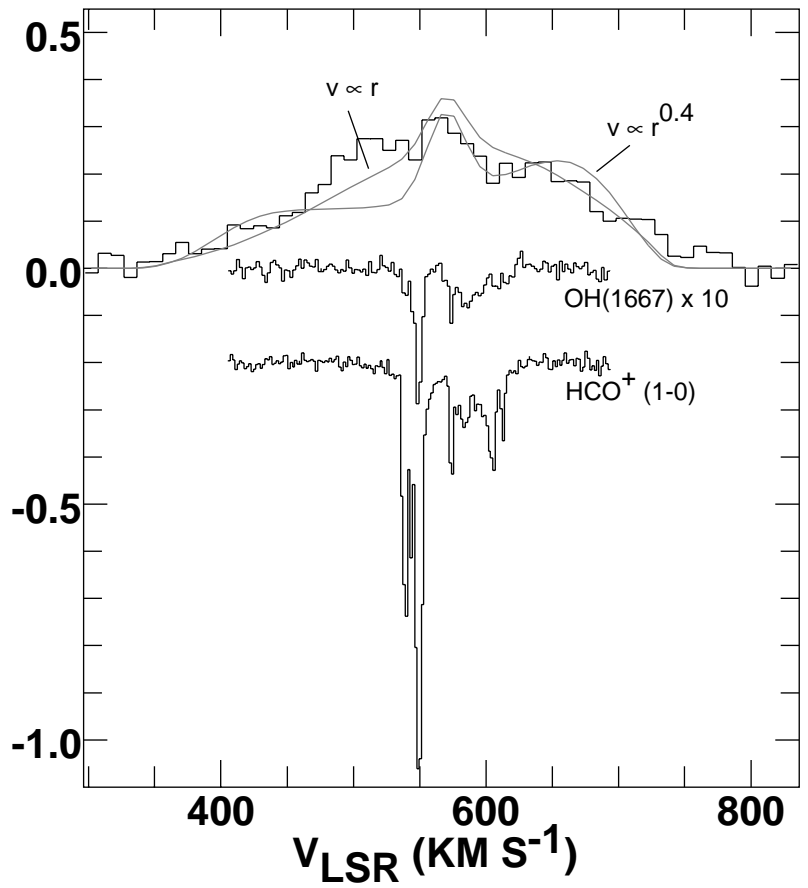

Fig. 6. $\mathrm{CO} J=3-2$ emission, $1667 \mathrm{MHz} \mathrm{OH}$ (van Langevelde et al. 1995) and $89 \mathrm{GHz} \mathrm{HCO}^{+}$absorption (Wiklind \& Combes 1997), and two model profiles. The form of the absorption spectra is line/continuum-1 and the $\mathrm{OH}$ profile has been multiplied by a factor 10 . Shown at top are the observed central (but slightly eccentric) emission profile (histogram) and two models of it, including contributions from the dust lane and the cnd, for the $v \propto r^{0.4}$ and $v \propto r$ power-law rotation curves

observed emission blueward of $540 \mathrm{~km} \mathrm{~s}^{-1}$ is not explained in any dust lane model: nor is this emission likely to have been affected by absorption. It really does appear that this emission cannot be explained except by the cnd. We tried adding non-circular velocities to the $v \propto r^{0.4}$ cnd model, but without success in this regard. The ability of the $v \propto r$ model to fit the data is only harmed by the addition of non-circular motions, which tend to make the profiles more horned, like those of the more rapidlyrising rotation laws. Given the lack of any compelling need to incorporate non-circular motions in cnd models to accomodate the red-shifted (or other) absorptions toward the nucleus, we find that the present observations are not well-suited to determination of any deviations from pure rotation.

Although it is a more obvious failure to leave highvelocity emission unexplained in a cnd model, it is in fact the emission nearer the systemic velocity which most profoundly shapes the picture of the cnd which is derived from data such as ours. It is an unfortunate paradox that only the less plausible kinematical model accounts so naturally for all of the observed emission which seemingly arises in the cnd.

\subsection{Contributions from material in the dust lane outside the cnd}

In order to understand the nature of the expected contribution from the outlying portions of the dust lane seen in projection, which provides the bulk of the emission in our dataset more than 1 or 2 pixels from from the nucleus, we constructed warped, twisted gas disks such as were described by Nicholson et al. (1992) for $\mathrm{H} \alpha$. In general we find that they are more much more nearly right than wrong, confirming the identification of $\mathrm{CO}$ and $\mathrm{H} \alpha$, but are not well-tuned. We believe that this may have resulted from low $\left(36 \mathrm{~km} \mathrm{~s}^{-1}\right)$ spectral resolution in the $\mathrm{H} \alpha$ data. For instance, the line splitting seen all along the strip at $\alpha=-10^{\circ}$ in Fig. 2 is not reproduced by the model except at larger angular separations, where it could clearly be seen in the $\mathrm{H} \alpha$ data.

One simple adjustment to the models of $\mathrm{H} \alpha$ emission which fixes much of the problem with the CO kinematics is to make the position-angle twist of the disk much bigger. As an example, we constructed a model in which the variation of inclination with radius is the same as shown in Fig. 9c of Nicholson et al. (1992), but the position angle increases from $80^{\circ}$ to $150^{\circ}$ over the inner $1475 \mathrm{pc}$ radius, rather than from $90^{\circ}$ to $132^{\circ}$ as in their Fig. 9a. The functional form of the variation in position angle is parabolic. Figure 7 shows the position-velocity diagrams from such a model overlaid on the gray-scales of Fig. 2. The model does a quite good job for those strips with $\alpha \lesssim 20^{\circ}$ but misses some of the gas at velocities above the systemic velocity, at negative offsets from the center, for $\alpha=20-50^{\circ}$; in this respect the model is neither better nor worse than that of Nicholson et al. (1992). We did not succeed in improving the fit in that portion of the data despite much work. Figure 8 shows how this model is situated with respect to the region observed, although, for clarity, only its lowest (i.e. smallest $|z|$ ) vertical portions are outlined. Clearly, any mapping program should not confine itself too narrowly along a single assumed long axis of the gas distribution.

The present observations sample the emission completely only for gas at radii $r \lesssim 1000 \mathrm{pc}$ and are not very sensitive at all to emission from gas at $r \gtrsim 1600 \mathrm{pc}$. The model represented in Fig. 7 has an inner radius of 650 pc; there is no doubt that the $\mathrm{CO}$ and $\mathrm{H} \alpha$ have a substantial central gap, as long as the rotation velocity at the inner edge of the model is not much smaller than the terminal velocity observed in the dust lane, $\approx 240 / \sin i \mathrm{~km} \mathrm{~s}^{-1}$ (Nicholson et al. 1992). A higher circular velocity at the inner edge would require a larger hole.

We attempted to taper the radial variation of the gas density to fit the data more precisely but found this difficult given the limited region we observed. The model shown in Fig. 7 has a doubled surface density for $r>$ $1100 \mathrm{pc}$, but this is probably an artifact of an imperfect fit. We got a somewhat better fit if the scale height of the gas increased outward, perhaps even greatly so, but such 


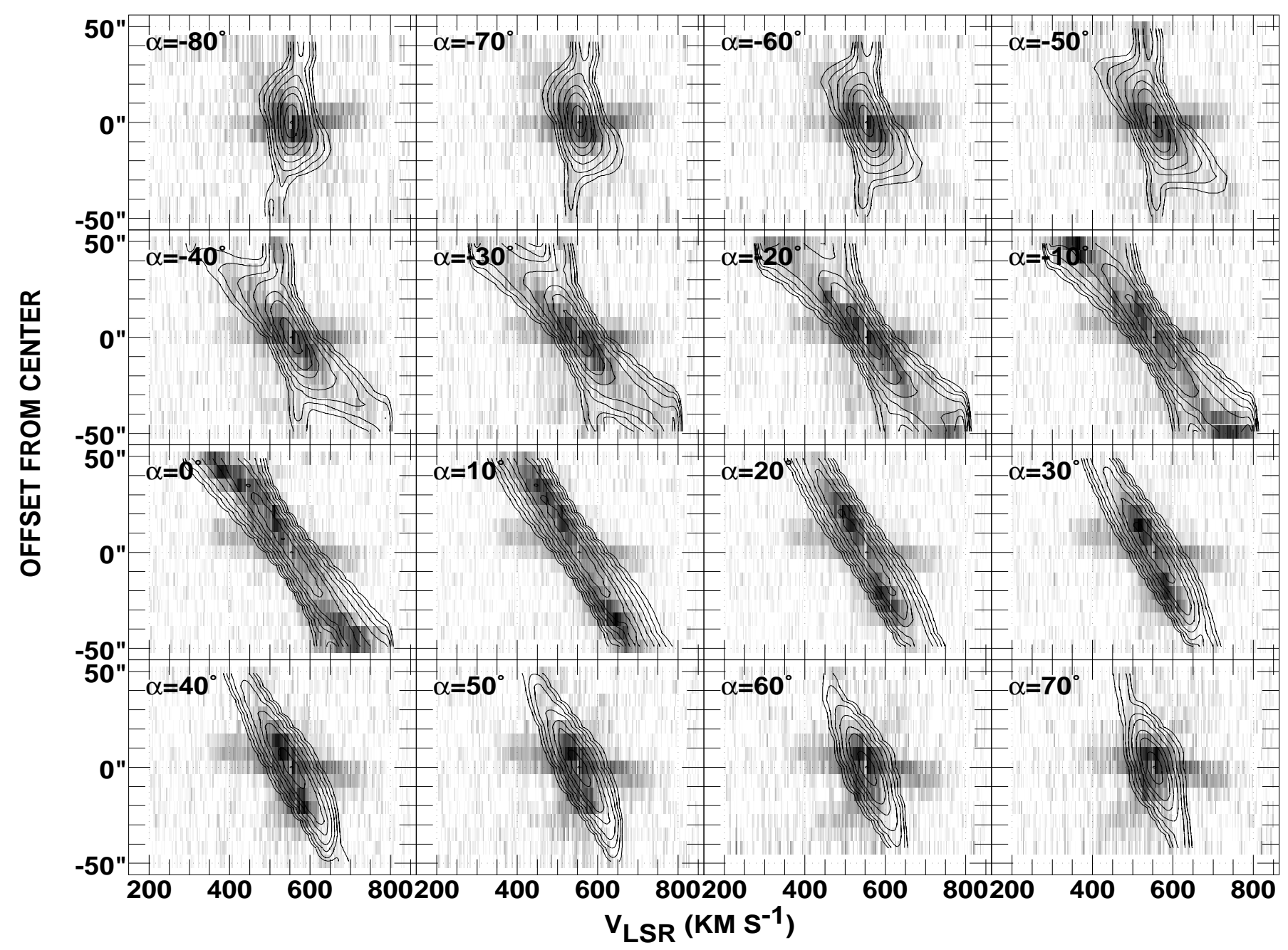

Fig. 7. Position-velocity diagrams formed from a more sharply twisted variant of the H $\alpha$ model of Nicholson et al. (1992), projected on the data of Fig. 2. The position angle on the sky is $\alpha+116.5^{\circ}$ and positive displacements are to the left of the grid's midpoint as shown in Fig. 1

inferences really require more extended mapping than we accomplished.

\section{Summary}

We mapped the central regions of the Cen A dust lane in the $J=3-2$ line of CO using $7^{\prime \prime}$ spacings of the $14^{\prime \prime}$ beam of the JCMT, affording somewhat better spatial resolution than has been previously available (a factor two in beam area). Nonetheless, conclusions regarding the structure and kinematics of the molecular circumnuclear disk are maddeningly model dependent, even to the extent that we cannot say definitively whether it was spatially resolved.

The simplest and best-fitting model we were able to construct of the emission ascribable to the cnd (some of which occurs quite close to the systemic velocity) is of a thin, radially-uniform, plane-parallel stratified disk of radius $168 \mathrm{pc}\left(10^{\prime \prime}\right.$; the assumed distance is $\left.3.5 \mathrm{Mpc}\right)$ and vertical dispersion $\sigma_{z}=47 \mathrm{pc}$, seen nearly edge-on (incli- nation $85^{\circ}$ ) in position angle $138.5^{\circ}$ on the sky (perpendicular to the base of the radio jet), in solid-body rotation having a speed of $195 \mathrm{~km} \mathrm{~s}^{-1}$ at its tip. In this case, the cnd was at least partly resolved, and the maximum rotation speed, edge-on geometry, position angle, etc. are fairly well constrained by the observations. In this model, CO emission extends to fairly small radii and the distribution is really a disk, not a narrow ring.

Dynamically plausible models having a more rapidlyrising rotation curve $v \propto r^{0.4}$ (which is a fair approximation to what is actually observed in $\mathrm{H}_{2}$ ) fit the data somewhat more poorly, but, if some emission nearer the systemic velocity is ignored (it cannot obviously arise in the dust lane), perhaps acceptably. In this case, although the best-fitting disk radius is somewhat larger, $190 \mathrm{pc}$, the luminosity or surface density in the model must have a strong radial taper such that its full width at halfmaximum $190 \mathrm{pc}$ or $12.5^{\prime \prime}$ is slightly less than the $14^{\prime \prime}$ beam. In this case, the model properties are not so well constrained, although a rotation speed of $170-195 \mathrm{~km} \mathrm{~s}^{-1}$ 


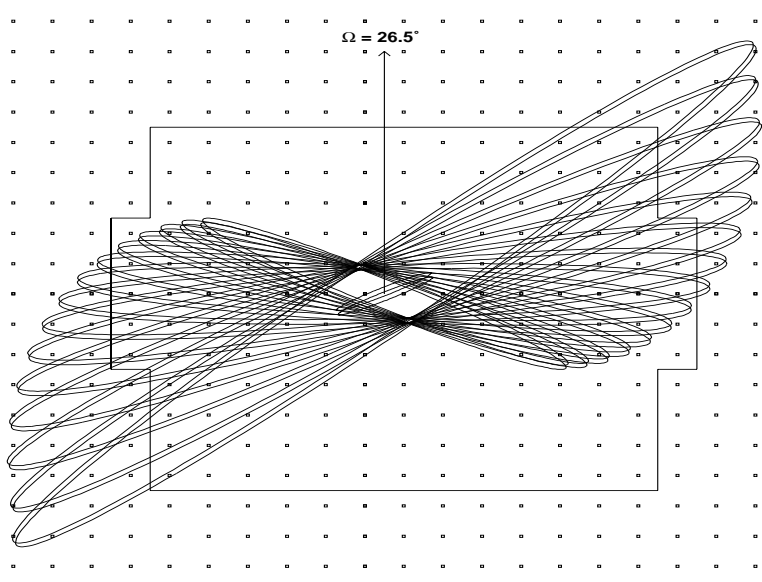

Fig. 8. Projection of the cnd and dust lane models on the observed map grid. Pairs of rings separated vertically by only $16 \mathrm{pc}$ are shown at radii $168 \mathrm{pc}$, and $650,710,770 \mathrm{pc}, \ldots$ The region sampled in this work, with the map grid marked, is outlined. The vertical axis is actually aligned along position angle $\Omega=26.5^{\circ}$ on the sky

at the outer edge of the cnd is still required and the geometry must be more nearly that of a full disk than a narrow ring.

In fact, there is no substitute for very high spatial resolution and the structure of the cnd really awaits either sub$\mathrm{mm}$ interferometry or mapping in $\mathrm{H}_{2}$ in the near-infrared. In the meantime, our larger-scale $\mathrm{CO}$ observations of gas in the dust lane do suffice to point out some minor inadequacies of the standard kinematic model of the dust lane based on $\mathrm{H} \alpha$ data, whose spectral resolution was too low to discern the true spatial extent of some line splittings. Knowledge of contributions from the dust lane to CO line profiles observed near nucleus will continue to be importance in interpreting the behaviour of gas in the cnd.

Acknowledgements. I am grateful to Goeran Sandell who took the data and to Remo Tilanus who helped in many ways. Observing for an earlier version of this project was begun in 1988 May with Thijs van der Hulst, who made three trips to the telescope in the years 1988-1990 before sensibly deciding to re-dedicate himself to $\lambda 21 \mathrm{~cm}$ work. I was assisted by $\mathrm{W}$. Butler Burton who made one trip in 1995, during which he at least found on the internet a much-needed source of library ladders. Walter Jaffe gave many helpful comments on the dynamics and core structure of elliptical galaxies. Frank Israel was a constant source of encouragement in finishing this work. Thanks to Françoise Combes and Huib van Langevelde for providing their absorption spectra in digital form. Close reading by the referee improved the paper.
The National Radio Astronomy Observatory is a facility of the National Science Foundation, operated by AUI, Inc. under a cooperative agreement. The JCMT is operated by the Joint Astronomy Centre on behalf of PPARC for the UK. The Netherlands Organization for Scientific Research, and the National Research Council of Canada.

\section{References}

Baade, W., \& Minkowski, R. 1954, ApJ, 119, 215

Bland, J., Taylor, K., \& Atherton, P. D. 1987, MNRAS, 228, 595

Eckart, A., Wild, W., \& Ageorges, N. 1999, ApJ, 516, 769

Faber, S. M., Tremaine, S., Ajhar, E. A., et al. 1997, AJ, 114, 1771

Ferrarese, L., van den Bosch, F. C., Ford, H. C., Jaffe, W., \& O'Connell, R. W. 1994, AJ, 108, 1598

Gebhardt, K., Richstone, D., Ajhar, E. A., et al. 1996, AJ, 112, 105

Hawarden, T. G., Sandell, G., Matthews, H. E., et al. 1993, MNRAS, 260, 844

Israel, F. P. 1992, A\&A, 265, 487

Israel, F. P., van Dishoeck, E. F., Baas, F., de Graauw, T., \& Phillips, T. G. 1991, A\&A, 245, L13

Israel, F. P., van Dishoeck, E. F., Baas, F., et al. 1990, A\&A, 227,342

Lauer, T. R., Ajhar, E. A., Byun, Y., et al. 1995, AJ, 110, 2622

Lauer, T. R., Faber, S. M., Tremaine, S., et al. 1997, in ASP Conf. Ser. 116: The Nature of Elliptical Galaxies; 2nd Stromlo Symposium, 113

Liszt, H. S. 1992, AJ, 104, 563

Marconi, A., Capetti, A., Axon, D., et al. 2001, ApJ, 549, 915

Marconi, A., Schreier, E. J., Koekemoer, A., et al. 2000, ApJ, 528,276

Mirabel, I. F., Laurent, O., Sanders, D. B., et al. 1999, A\&A, 341,667

Nicholson, R. A., Bland-Hawthorn, J., \& Taylor, K. 1992, ApJ, 387,503

Phillips, T. G., Ellison, B. N., Keene, J. B., et al. 1987, ApJ, 322, L73

Quillen, A. C., de Zeeuw, P. T., Phinney, E. S., \& Phillips, T. G. 1992, ApJ, 391, 121

Quillen, A. C., Graham, J. R., \& Frogel, J. A. 1993, ApJ, 412, 550

Roberts, M. S. 1970, ApJ, 161, L9

Rydbeck, G., Wiklind, T., Cameron, M., et al. 1993, A\&A, 270, L13

Tubbs, A. D. 1980, ApJ, 241, 969

van Gorkom, J. H., van der Hulst, J. M., Haschick, A. D., \& Tubbs, A. D. 1990, AJ, 99, 1781

van Langevelde, H. J., van Dishoeck, E. F., Sevenster, M. N., \& Israel, F. P. 1995, ApJ, 448, L123

Wiklind, T., \& Combes, F. 1997, A\&A, 324, 51 